\title{
Preservation of Leptospiras by Liquid-Nitrogen Refrigeration
}

\author{
A. D. ALEXANDER, E. F. LESSEL, L. B. EVANS, E. FRANCK, and S. S. GREEN ${ }^{1}$
}

Department of Veterinary Microbiology, Division of Veterinary Medicine, Walter Reed Army Institute of Research, Washington, D.C. 20012, and American Type Culture Collection, Rockville, Maryland 20852

\begin{abstract}
The viability and virulence of an 8-day-old culture of a hamster-virulent strain of Leptospira interrogans serotype canicola in Stuart's medium were successfully preserved by the use of liquid nitrogen for freezing and storage. Glycerol was added to the culture to give a final $10 \%$ concentration. The culture was rapidly cooled at a rate of $65 \mathrm{C}$ degrees per min from $0 \mathrm{C}$ to $-130 \mathrm{C}$ and then stored in the vapor portion of the liquid-nitrogen refrigerator. Prior to freezing, the viability count and $\mathrm{LD}_{50}$ titer for hamsters were $1.2 \times 10^{8}$ per ml and $10^{-7.2}$, respectively. One day after freezing, the viability and virulence values were reduced 10- to 100-fold but remained essentially unchanged thereafter over a 5 -year observation period. The serological properties of the strain did not appear to be altered after prolonged storage. All except 9 of 103 additional cultures of diverse $L$. interrogans serotypes grown in either Stuart's medium or in a Tween-80 albumin medium were successfully preserved by liquid-nitrogen refrigeration over a 7 - to 38-month observation period with glycerol as a cryoprotective agent and a controlled rate of freezing of 35 or $60 \mathrm{C}$ drop per min. Failures to recover leptospiras could be attributed to either the presence of contaminating microorganisms or the sparse concentration of organisms in the original preparation. The viability of thawed cultures persisted for 7 days when held at room temperature. The feasibility of storage of stock leptospiral cultures by use of liquid-nitrogen refrigeration was demonstrated.
\end{abstract}

Leptospiras are ordinarily maintained in laboratories by regular transfers in appropriate media at 3-week to 3-month intervals (1). Virulence of leptospiral strains can be ascertained only by sequential or periodic passages in laboratory animals. The conventional freezing procedures at temperatures of -20 to -78 $C$ used for preserving other microorganisms have not proved to be consistently successful for long-term preservation of leptospiras (3, 14, 15). Attempts to preserve leptospiras by freeze-drying have rarely been successful $(4,5$; Schubert and Sulzer, Bacteriol. Proc., 1970, p. 20). Exceptional and promising results were reported by Otsuka and Manako (6), who succeeded in preserving viability in 30 strains by freeze-drying heavy concentrations of organisms in a leptospiral culture milieu fortified with a peptone-starch adjuvant. Under the described conditions of freeze-drying and stor-

\footnotetext{
${ }^{1}$ Present address: Experimental Immunology Division, Clinical Medical Sciences Department, Naval Medical Research Institute, Bethesda, Md. 20014,
}

age, all strains were recovered in culture after a 2-year observation time.

In recent years, the need to develop a satisfactory means for long-term preservation has increased because of: (i) the continuous disclosure of new serotypes and consequent increase in number of cultures to be maintained, (ii) requirements for a large battery of strains for use as antigens in serological tests, and (iii) requirements to maintain and standardize strain virulence for basic as well as applied research. The use of liquid nitrogen for freezing and storing leptospiras appears to afford a practical means for preserving their viability and virulence. Resseler and van Riel (8), Torney and Bordt (12), and Stalheim (9) preserved leptospiras by using either glycerol or dimethyl sulfoxide as a protective additive to cultures which were frozen and stored in liquid nitrogen. All of 10 strains tested by Resseler and van Riel (8) were viable after 1 year of storage. Torney and Bordt (12) found that the viability and virulence titers of cultures dropped 1 to 2 logs (base 10) after freezing, but thereafter re- 
mained constant when tested over a 21 - to 24month period of storage. Stalheim (9) obtained similar results with a Leptospira interrogans serotype pomona culture containing added glycerol $(10 \%, \mathrm{v} / \mathrm{v})$ over a 6 -month period of storage.

Qualitative and quantitative observations over a 5-year period on the use of liquidnitrogen refrigeration to preserve leptospiras are presented.

\section{MATERIALS AND METHODS}

Bacterial strain. A hamster-virulent strain (MoultonATCC 23606) of $L$ interrogans serotype canicola was used for quantitative evaluation of its virulence and viability over a 5 -year period of storage in a liquid-nitrogen refrigerator. The strain was isolated in 1952 from the blood of a sick dog. Over the years, its virulence was maintained and checked by periodic passages in hamsters.

Freezing. The preparation used for freezing was the pooled contents of 40 tubes of 8-day-old cultures in Stuart medium containing $10 \%$ rabbit serum (11). Each tube of culture contained 5 to $7 \mathrm{ml}$ of medium and was inoculated with $1 \mathrm{drop}$ (ca. $0.05 \mathrm{ml}$ ) of blood from moribund hamsters experimentally infected with the Moulton strain. All cultures comprising the pool had good growth of leptospiras. The pooled culture was ascertained to be free of contaminating microorganisms by cultural tests on blood-agar and thioglycolate broth. Sterile glycerol was added to the culture to provide a final $(\mathrm{v} / \mathrm{v})$ concentration of $10 \%$. The pooled culture was distributed in $0.5-\mathrm{ml}$ amounts in $1.2-\mathrm{ml}$ ampoules which were heat sealed, placed in a controlled-rate freezer (Linde Biological Freezer, $\mathrm{BF}-4$ ), and cooled at a rate of $60 \mathrm{C}$ degrees per min (from 0 to $-130 \mathrm{C}$ ) by exposure to liquid-nitrogen vapor. The frozen cultures were stored in a vapor phase cannister of a liquid-nitrogen refrigerator $(-168$ C) at the American Type Culture Collection (ATCC), Rockville, Md.

Viability and virulence determinations. Viability and virulence determinations were made on the pooled culture prior to freezing and on frozen samples obtained 1 day after freezing and at periodic intervals thereafter up to a 5-year observation period. Samples were rapidly thawed by immersion in a $37 \mathrm{C}$ water bath, pooled, and serially diluted by half logarithmic (base 10) increments with Stuart medium. The concentration of viable leptospiras was determined by microscopic counts with the use of a Petroff-Hauser counting chamber and also by subculturing each dilution in three or five tubes of Fletcher medium (13). Each tube, containing approximately $5 \mathrm{ml}$ of medium, was inoculated with either $0.1 \mathrm{ml}$ or $0.5 \mathrm{ml}$ of diluted culture. Cultures were incubated at $30 \mathrm{C}$ and examined for presence of leptospiras at 1- or 2-week intervals for up to 6 weeks. The Reed-Muench method was used for viability quantitation (12). Titers were expressed as the $\log _{10}$ tube inoculating dose $_{50}$ $\left(\mathrm{TID}_{50}\right)$ per milliliter. The microscopic counts were made on a suitable dilution of culture to provide a concentration of 12 to 25 leptospiras per intermediate-size square $\left(0.04 \mathrm{~mm}^{2}\right)$ of the counting chamber. Duplicate counts were made, each count involving at least five intermediate squares. Only motile organisms were counted.

Virulence titrations were done in 3- to 4-week-old hamsters with 5 or 10 animals used for each dilution. Inocula were given intraperitoneally in $0.5-\mathrm{ml}$ doses. After inoculation, animals were observed for 16 days. The presence of leptospiras in dead or moribund animals was determined by examining blood or liver suspensions by microscopic or cultural or both procedures. The $\mathrm{LD}_{5_{0}}$ was calculated by the ReedMuench formula (7).

Qualitative studies were done on 75 strains comprising 51 pathogenic and 3 "saprophytic" serotypes. The strains were grown for 4 to 8 days in one or two tubes of Stuart medium, supplemented with glycerol $(10 \%)$, and distributed in ampoules. The ampoules were sealed and then frozen and stored as described. At various times up to 38 months, samples of frozen cultures were rapidly thawed in a $37 \mathrm{C}$ water bath, examined microscopically for the presence of motile organisms, and cultured in at least two tubes of Fletcher medium. In some trials, Stuart and modified Ellinghausen Albumin Fatty Acid Medium (EMJH) were also used for cultural tests (13).

Additional tests were done on 1 "saprophytic" and 27 pathogenic strains, which were grown in EMJH medium for 4 to 7 days. Glycerol was then added to give a final concentration of $10 \%$. In this series, the cultures were distributed in $1-\mathrm{ml}$ amounts in screwcapped vials ( 15 by $45 \mathrm{~mm}$ ). The cultures were placed directly in the vapor phase of the liquid-nitrogen refrigerator. The rate of cooling under these conditions was determined to be $35 \mathrm{C}$ degrees per min from 0 to $-130 \mathrm{C}$. Freezing of 28 cultures in EMJH medium was done at two different times 4 months apart on 7 and 21 strains, respectively. The viability of the stored cultures in EMJH medium was determined by cultural tests in Fletcher medium over a 7- to 16-month period of storage.

Microscopic agglutination and agglutinin-adsorption tests were performed according to described procedures $(1,2)$.

\section{RESULTS}

Data on the viability and virulence of the Moulton strain of $L$. interrogans serotype canicola over a 5-year observation period are summarized in Table 1. Prior to freezing, the viability count and culture titer $\left(\right.$ TID $\left._{5_{0}}\right)$ were $1.2 \times 10^{8}$ per ml and $10^{-8.1}$, respectively. The $\mathrm{LD}_{5} 0$ titer for hamsters was $10^{-7.2}$. At 1 day after freezing, the viability values were decreased by 1.2 to $2.0 \operatorname{logs}$ (base 10 ) and the virulence titer by 1.8 logs. After 5 years of storage, no further decrease in viability or 
virulence was evident. The observed variations in microscopic counts and in the viability and virulence titers obtained at different test intervals were not surprising. They may well reflect variables introduced by the uncontrolled method of thawing and by differences in techniques of various personnel conducting tests. Furthermore, variations in the growthsupporting quality of different lots of media could figure in TID $_{50}$ titer differences. Variations in $L^{2} D_{5}$ values may also be related to relative susceptibility of hamsters used at different test periods. The 2-year test sample was removed from the liquid $\mathrm{N}_{2}$ refrigerator at the ATCC, thawed, and then mailed to the Walter Reed Army Institute of Research (WRAIR). Two days after mailing, the ampoules were opened, and the contents were pooled and tested. This delayed handling had no apparent deleterious effect on viability and virulence.

Organisms recovered from a terminal dilution $\left(10^{-7.5}\right)$ of the sample stored for 5 years were serologically indistinguishable from a prefrozen culture on the basis of cross-agglutination and agglutinin-adsorption tests.

Approximately 2 years after strain Moulton was frozen, storage of a relatively large number of leptospiral cultures by liquid-nitrogen refrigeration was initiated. These efforts were prompted by the promising results obtained in preserving the Moulton strain. Test findings on viability of strains cultured in Stuart and EMJH media are shown in Table 2. Leptospiras were successfully preserved in either Stuart or EMJH medium each of which contained $10 \%$ glycerol. Recovery of leptospiras succeeded when cultures in the respective media were cooled at rates of 60 or $35 \mathrm{C}$ degrees per min (from 0 to $-130 \mathrm{C})$. The failure to recover in culture 9 of the 75 strains grown in Stuart medium could be attributed in seven instances to the presence of contaminating microorganisms. The other two strains had sparse growth when frozen.

From a practical viewpoint for maintenance and distribution of leptospiras by culture collections, it was important to determine if the viability of a thawed culture could persist over a reasonable period of time needed for mail delivery. For this purpose, multiple ampoules of the frozen Moulton strain at ATCC were thawed and then mailed to WRAIR where they were received the next day and held at room temperature for 6 additional days. They were then opened, and their contents were pooled and diluted to $10^{-5}$. Each dilution was subcultured to Fletcher medium; the $10^{-1}$ and $10^{-2}$ dilutions were inoculated into hamsters by

TABLE 1. Viability and virulence of Leptospira interrogans serotype canicola strain Moulton after storage in the vapor phase $(-168 \mathrm{C})$ of a liquid-nitrogen refrigerator

\begin{tabular}{lccc}
\hline Preservation & $\begin{array}{c}\text { Microscopic } \\
\text { time }\end{array}$ & $\begin{array}{c}\text { Viability } \\
\text { titer } \\
\left(-\log _{10} \text { o }\right. \\
\left.\text { cID }_{50}\right)\end{array}$ & $\begin{array}{c}\text { Virulence } \\
\text { titer } \\
\left(-\log _{10}\right. \\
\left.\text { LD }_{50}\right)\end{array}$ \\
\hline Pre-frozen & $1.2 \times 10^{8}$ & 8.1 & 7.2 \\
1 day & $8.1 \times 10^{6}$ & 6.1 & 5.4 \\
1 month & $1.1 \times 10^{7}$ & 6.3 & 4.5 \\
7 months & $1.1 \times 10^{7}$ & 7.1 & 5.2 \\
1 year & $1.5 \times 10^{7}$ & 5.4 & 4.7 \\
2 years & $2.0 \times 10^{7}$ & Not done & 6.2 \\
4 years & $8.6 \times 10^{6}$ & 6.2 & 5.7 \\
5 years & $8.0 \times 10^{6}$ & 6.4 & 5.0 \\
\hline
\end{tabular}

$a$ Thawed sample was mailed from ATCC, Rockville, Md., to WRAIR, Washington, D.C., and opened and tested 2 days after removal from the liquid-nitrogen refrigerator.

TABLE 2. Recovery of strains of Leptospira after storage in the vapor phase (-168 C) of a liquid-nitrogen refrigerator

\begin{tabular}{|c|c|c|c|c|c|c|c|c|c|}
\hline \multirow[b]{2}{*}{ Culture medium $^{a}$} & \multirow{2}{*}{$\begin{array}{l}\text { No. of } \\
\text { strains }\end{array}$} & \multicolumn{7}{|c|}{ No. viable/no. examined after storage (months ${ }^{b}$ ) } & \multirow{2}{*}{$\begin{array}{c}\text { No. viable/ } \\
\text { total no. } \\
\text { of strains }\end{array}$} \\
\hline & & 7 & $11-12$ & 16 & 21 & 23 & $25-26$ & 38 & \\
\hline $\begin{array}{l}\text { Stuart ....... } \\
\text { EMJH (Tween } \\
80 \text {-albumin) }\end{array}$ & $\begin{array}{l}75 \\
28\end{array}$ & $21 / 21$ & $\begin{array}{l}15 / 16 \\
18 / 18\end{array}$ & $7 / 7$ & $28 / 30$ & $15 / 15$ & $46 / 48$ & $51 / 59$ & $\begin{array}{l}66 / 75^{c} \\
28 / 28\end{array}$ \\
\hline
\end{tabular}

${ }^{a}$ Cultures in Stuart medium were cooled in a "controlled-rate freezer" $(-60 \mathrm{C}$ degrees per min from 0 to $-130 \mathrm{C}$ ), whereas cultures in EMJH were placed directly in the vapor of the liquid-nitrogen refrigerator $(-35 \mathrm{C}$ degrees per $\min$ ).

${ }^{b}$ All strains were tested at least once. Strains which were not viable by cultural tests were retested at one or more testing periods.

${ }^{c}$ Nine strains were culturally negative on one or more examinations; seven of these had contaminating microorganisms, the other two had sparse grow th when frozen. 
using previously described methods. Leptospiras were recoverable in culture from all dilutions tested. The two dilutions tested provoked fatal infections in hamsters. The storage of stock cultures of leptospiras at national culture collections appeared to be practical. Therefore, between 7 November 1967 and 15 February 1968, the 14 most frequently used serotype strains of leptospiras were deposited and stored at the ATCC in the manner described above. Up to 30 July 1971, 48 cultures of leptospiras representing 8 strains were distributed by the ATCC, and only two complaints of nonviability were received. However, a check of cultures from the batches from which the reportedly nonviable cultures originated showed that the cultures in stock are viable.

\section{DISCUSSION}

Our findings confirm and extend those of Resseler and van Riel (8) and Torney and Bordt (12) who reported that leptospiras can be preserved successfully by the use of liquidnitrogen refrigeration.

At first, glycerol was selected as a cryoprotective agent because it has been commonly used as an ingredient in leptospiral media (11). Furthermore, in preliminary qualitative tests, motile leptospiras could still be found in cultures in Stuart medium after the addition of glycerol to 5 or $10 \%$ final concentration and a 6-day holding period at room temperature. Exposure of leptospiras to glycerol concentrations of 15 and $20 \%$ resulted in a loss of motility of all leptospiras. Linscott and Boak (4) previously reported the immobilizing effect of $15 \%$ glycerol on leptospiras. They further reported that $5 \%$ glycerol was less toxic but inhibitory. The possible grow th-inhibitory effect of $10 \%$ glycerol did not obviate its usefulness as a cryoprotective agent for leptospiras grown in either a $10 \%$ serum medium (Stuart) or in the EMJH medium which contains approximately $1 \%$ bovine albumin and $0.1 \%$ polysorbate (Tween 80 ) in lieu of serum. The relative advantages of these two media in supplementing the cryoprotective properties of glycerol or in limiting its growth-inhibiting effects were not determined. The successful recovery of leptospiras from thawed cultures in Stuart medium after delayed holding periods under experimental as well as applied conditions was further proof that glycerol in $10 \%$ concentration was an adequate cryoprotective agent. Tests subsequently conducted by Stalheim (9) also affirmed the efficacy of $10 \%$ glycerol as a cryoprotective agent. The quantitative viability data in this study were comparable to those of Torney and Bordt (12), although their cooling rates ranged from 1 to 5 $\mathrm{C}$ degrees per min, whereas we used cooling rates of 35 or $60 \mathrm{C}$ degrees per min. Freezing of cultures resulted in a 1 - to $2-\log$ decrease in viability titer in both studies. The preservation of leptospiras could probably be enhanced by tests to establish the optimum cooling rate and optimum level of glycerol or other cryoprotective agent.

Leptospiras were preserved equally well in the vapor-phase portion $(-168 \mathrm{C})$ and in the liquid phase $(-196 \mathrm{C})$ of a liquid-nitrogen refrigerator. When stored at temperatures above $-100 \mathrm{C}$, viable leptospiras have not been recovered consistently even when cryoprotective agents suitable at lower temperatures were used. Equivocal findings on the effects of storage temperatures in the range above $-79 \mathrm{C}$ have been observed with other microorganisms (5). We prefer to use the vapor portion of the liquid-nitrogen refrigerator primarily because the explosion hazard in the thawing of cultures is reduced and secondly because it allows the use of screw-capped vials in lieu of hermetically sealed ampoules to contain the culture. The screw-capped vials do not require a special sealing process, they are safer and easier to handle, and are sturdier than sealed ampoules.

The effect of age of culture on the survival of leptospiras after freezing and storage by liquidnitrogen refrigeration was not determined. The age of the cultures used in this study at the time of freezing ranged from 4 to 8 days. Torney and Bordt (12) froze 7- to 9-day-old cultures. Thus it appears that cultures in the late logarithmic phase of growth (i.e., 4 to 9 days for leptospiras) may be used for preservation by liquid-nitrogen refrigeration. Otsuka and Manako (6) reported that 7- to 14-day-old cultures had a high survival rate after freeze drying, whereas those 21 days or older had poor survival. Thus it would appear that stationary-phase leptospiras would similarly be less likely to survive freezing and storage in liquid-nitrogen refrigerators.

A critical factor for the successful preservation of leptospiras in liquid nitrogen is cell concentration. In this study, failures to recover leptospiras culturally after long-term preservation, other than those related to bacterial contamination, were attributable to sparse concentration of leptospiras at the time of freezing. Concentration of cells was also found to be a critical factor for successful recovery of leptospiras preserved by freeze-drying (6).

Our selection of the specific conditions for 
freezing and storing leptospiras was admittedly empirical and was based on the cryobiological methods and principles applied successfully to the preservation of other microorganisms. Nevertheless, our methods provided an expedient means for the long-term preservation of leptospiras. There was no evidence that the serological properties of a virulent leptospiral strain were altered after long-term preservation in the manner described above.

Critical factors for preserving leptospiras in a viable and stable condition by low-temperature freezing and storage are: (i) the type and concentration of cryoprotective agent added to the culture; (ii) the cooling rate; (iii) the storage temperature; and (iv) the concentration of organisms. The age of the culture at the time of freezing is also probably an important factor.

\section{LITERATURE CITED}

1. Alexander, A. D. 1970. Leptospira, p. 224-250. In J E. Blair, E. H. Lennette, and J. P. Truant (ed.), Manual of clinical microbiology. American Society for Microbiology, Bethesda, Md.

2. Alexander, A. D., L. B. Evans, H. Jeffries, C. A. Gleiser, and R. H. Yager. 1954. Serologic characterization of the Fort Bragg leptospire. Proc. Soc. Exp. Biol. Med. 86:405-408.

3. Coghlan, J. D., W. H. R. Lumsden, and G. J. C. McNeillage. 1967. Low-temperature preservation of Leptospira, preliminary communication. J. Hyg. 65:373-379.

4. Linscott, W. D., and R. A. Boak. 1960. Protective action of glycerol in the freezing of leptospirae. J. Bacteriol. 80:573-574.

5. Meryman, H. T. 1963. Preservation of living cells. Fed. Proc. 22:81-89.

6. Otsuka, S., and K. Manako. 1961. Studies on the preservation of leptospirae by freeze-drying. Jap. J. Microbiol. 5:141-148.

7. Reed, L. J., and H. Muench. 1938. A simple method of estimating fifty percent end points. Amer. J. Hyg. 27:493-497.

8. Resseler, R., and J. van Riel. 1966. Conservation des leptospires apres refroidissement ou lyophilisation. Ann. Soc. Belge. Med. Trop. 46:213-222.

9. Stalheim, O. H. V. 1971. Viable, avirulent Leptospira interrogans serotype pomona vaccine: preservation in liquid nitrogen. Appl. Microbiol. 22:726-727.

10. Stavitsky, A. B. 1945. Preservation of Leptospira icterohaemorrhagiae in vitro. J. Bacteriol. 50:118.

11. Stuart, R. D. 1946. The preparation and use of a single culture medium for leptospirae. J. Pathol. Bacteriol. 58:343-349.

12. Torney, H. L., and D. E. Bordt. 1969. Viability quantitation of leptospires after rapid and controlled rate freezing. Cryobiology 5:352-354.

13. Vera, H. D., and M. Dumoff. 1970. Culture media, 633-674. In J. E. Blair, E. H. Lennette and J. P. Truant (ed.), Manual of clinical microbiology. American Society for Microbiology, Bethesda, Md.

14. Weinman, D., and J. McAllister. 1947. Prolonged storage of pathogenic protozoa with conservation of virulence. Amer. J. Hyg. 45:102-121.

15. Wolff, J. W. 1960. Preservation of leptospires. In Leptospirae and Leptospirosis in Man and Animals. The Problem Sessions Series of the Polish Academy of Sciences 19:11-15. 